\title{
CONDITIONS FOR A TVS TO BE HOMEOMORPHIC WITH ITS COUNTABLE PRODUCT
}

BY

\section{WESLEY E, TERRY}

ABSTRACT. C. Bessaga has given conditions for a Banach space to be homeomorphic with its countable product. In this paper, we extend and generalize these results to complete metric topological vector spaces by using infinite dimensional techniques.

1. Introduction. Let $M$ be a topological vector space (TVS), and let $M^{\omega}$ denote its countable product. The hypothesis that $M$ is homeomorphic to $(\widetilde{\sim}) M^{\omega}$ is contained in several important theorems in infinite dimensional topology. For example, it is used in Henderson and Schori's paper [8]. In the following, we will establish equivalent conditions for a metric TVS $M$ to be homeomorphic to $M^{\omega}$.

If $d$ is a metric on a TVS $M$ we say that $d$ is translation invariant if $d(x+y, y)=d(x, 0)$ for all $x, y \in M$. We say that $d$ is strictly monotone if $0 \leq$ $t<s$ implies $d(t x, 0)<d\left(s_{n}, 0\right)$ for all nonzero $x \in M$.

Let $c_{0}$ be the Banach space consisting of sequences of real numbers such that the absolute values tend toward zero. The norm is the supremum norm. Let $l_{p}(p>0)$ be the collection of sequences of reals $\left\{x_{i}\right\}$ such that $\Sigma_{i=1}^{\infty}\left|x_{i}\right|^{p}<\infty$ with the quasi-norm being $\left\{\Sigma_{i=1}^{\infty}||^{p}\right\}^{1 / p}$. Finally, let $\left\{a_{a_{n}}\right\}_{a, n=1,2, \ldots}$ be a matrix of positive real numbers satisfying:

(1) $a_{1 n} \leq a_{2 n} \leq \cdots(n=1,2, \ldots)$, and

(2) $\sum_{n=1}^{\infty} a_{a n} / a_{a+1 n}<\infty(a=1,2, \cdots)$.

By $M\left(a_{a_{n}}\right)$, we mean the collection of all real sequences, $x=\left\{x_{i}\right\}$, such that $|x|_{a}=$ $\sup _{n} a_{a_{n}}\left|x_{n}\right|<\infty$ for all $a$. The metric on $M\left(a_{a_{n}}\right)$ is then just

$$
d(x, y)=\sum_{a=1}^{\infty} \frac{1}{2^{a}} \frac{|x-y|_{a}}{1+|x-y|_{a}} .
$$

Given $\left\{\left(M^{i}, d^{i}\right) \mid i=1,2, \ldots\right\}$, a collection of complete metric TVS's such that each metric is translation invariant and strictly monotone, and given one of the coordinate spaces $E$ listed above, we define $\Sigma_{E} M^{i}$ to be the set of sequences

Received by the editors August 16, 1972 and, in revised form, February 10, 1973. AMS (MOS) subject classifications (1970). Primary 46A45, 57 A20; Secondary 46A05.

Key words and phrases. Topological vector space, countable product, infinite dimensional Fréchet space, $Z$-set, strongly negligible. 
$\left\{x_{i}\right\}, x_{i} \in M^{i}$, such that $\left\{d^{i}\left(x_{i}, 0\right)\right\} \in E . \Sigma_{E} M^{i}$ has the topology induced from the metric $\rho_{E}\left(\left\{d^{i}(),\right\}, 0\right)$ where $\rho_{E}$ is the metric on $E$. It is easy to show that $\Sigma_{E} M^{i}$ is a metric TVS. We will use the notation $\Sigma_{E} M$ when all the spaces $\left(M^{i}, d^{i}\right)_{=}$ $(M, d)$ for some fixed pair $(M, d)$.

Bessaga has proven in $[5]$ that:

Theorem. Let $X$ be a Banach space with norm \|\| . If $X \cong \Sigma_{l_{1}} X$ (using $\|-\|$ for $d$ above), then $X \cong X^{\omega}$.

As a consequence of this theorem, Bessaga has shown that, for $\boldsymbol{X} \geq \boldsymbol{N}_{0}$, $l_{1}(x) \simeq l_{1}(x)^{\omega}$. Here $l_{1}(x)$ is the set of $\boldsymbol{x}$-sequences of reals, $\left\{x_{\lambda}\right\}_{\lambda \in C_{\text {ard }}(x)}$ with at most countably many nonzero coordinates and with $\Sigma_{\lambda \in \operatorname{Card}(\boldsymbol{K})}\left|x_{\lambda}\right|<\infty$. The norm is the obvious one.

Using entirely different techniques, we have proven the converse of this theorem, and, in fact, have shown

Theorem 0. Let $X$ be an infinite dimensional Banach space. Then $\Sigma_{l_{1}} X \cong$ $X^{\omega}$ using any equivalent norm on $X$.

We have also generalized this result to:

Theorem 1. Let $M$ be a complete metric TVS with translation invariant, strictly monotone metric $d$. Then $M \cong M^{\omega}$ if and only if botb $M \cong l_{1} \times M$ and $M \cong \Sigma_{l_{1}} M$.

Note that we are not assuming that $M$ is either locally convex or separable.

Remark 1. It was shown by R. D. Anderson [1] that all separable Fréchet (complete, locally convex, metric TVS) spaces are homeomorphic to $l_{1}$. Then, since every infinite dimensional Fréchet space $F$ clearly contains a closed, separable, infinite dimensional subspace $K$, we know that every infinite dimensional Fréchet space contains a closed subspace homeomorphic to $l_{1}$. We may apply the Bartle-Graves-Michael theorem to the quotient space $F / K$ to produce a homeomorphism between $F$ and $K \times F / K$ (see [5, Theorem 8.1]). Since $l_{1}$ is obviously homeomorphic to $l_{1} \times l_{1}$, we have:

$$
F \cong K \times F / K \cong l_{1} \times F / K \cong l_{1} \times\left(l_{1} \times F / K\right) \cong l_{1} \times F
$$

Therefore, every infinite dimensional Fréchet space $F$ satisfies $F \cong l_{1} \times F$ and, in particular, so does every Banach space.

Remark 2 As a consequence of Theorem 6.7 [9] and of a result of Eidelheit and Mazur [7] all complete metric TVS's have a translation invariant, strictly monotone metric. Thus, every complete metric TVS has a metric satisfying the hypothesis of Theorem 1 .

Now, the proof of Theorem 1 is similar in style to the Anderson-Bing paper [3]. We actually show that: 
(Step 1) $\quad l_{1} \times \Sigma_{l_{1}} M \simeq \Sigma_{l_{1}}\left[\left(\Sigma_{l_{1}} l_{1}\right) \times M\right]$

(Step 2)

$$
\simeq \Sigma_{l_{1}}\left[\left(\Sigma_{l_{1}} l_{1} \backslash(0\}\right) \times(M \backslash\{0\})\right]
$$

(Step 3)

$$
\cong\left[\left(\Sigma_{l_{1}} l_{1}\right)^{\omega} \backslash\left(\cup l^{i j}\right) \cup\left(\cup K^{i}\right)\right] \times[M \backslash\{0\}]^{\omega}
$$

(Step 4)

$$
\simeq\left(\Sigma_{l_{1}} l_{1}\right)^{\omega} \times M^{\omega}
$$

(Step 5)

$$
\cong M^{\omega} \text {, }
$$

where $l^{i j}=\left\{\left\{\eta^{k}\right\} \in\left[\Sigma_{l_{1}} l_{1}\right]^{\omega}: \pi_{j}\left(\eta^{i}\right)=0\right\}$ using $\pi_{j}$ to mean the projection onto the $j$ th coordinate of $\Sigma_{l_{1}} l_{1}$, and where

$$
K^{i}=\left\{\left\{r^{i}\right\} \in\left[\Sigma_{l_{1}} l_{1}\right]^{\omega}: \sum_{i=1}^{\infty} \frac{1}{1+\left|r^{i}\right|} \leq i\right\}
$$

using | to represent the norm on $\Sigma_{l_{1}} l_{1}$. The $K^{i}$ 's and $l^{i j}$ 's occur as technical difficulties, in that we construct a map which, when restricted to the complement of these sets, is a homeomorphism.

As a consequence of the proof of Theorem 1, we also have the following:

Theorem 2. Let $\left\{\left(M^{i}, d^{i}\right) \mid i=1,2, \ldots\right\}$ be a collection of complete metric TVS's such that eacb metric is translation invariant and strictly monotone. If $M^{j} \simeq l_{1} \times M^{j}$ for some $j$, then $\Sigma_{l_{1}} M^{i} \cong \Pi_{i=1}^{\infty} M^{i}$.

We may generalize these results to obtain:

Theorem 3. Let $E$ and $E^{\prime}$ be any two of the spaces $c_{0}, l_{p}(p>0), M\left(a_{a_{n}}\right)$. Let $M$ be a complete metric TVS with translation invariant, strictly monotone metric d. Then $M \cong M^{\omega}$ if and only if both $M \cong l_{1} \times M$ and $M \cong \Sigma_{E} M$. Also, if $\left\{\left(M^{i}, d^{i}\right) \mid i=1,2, \ldots\right\}$ is a collection of complete metric TVS's sucb that each $d^{i}$ is translation invariant and strictly monotone, and if $l_{1} \times M^{j} \cong M^{j}$ for some $j$, then $\Sigma_{E} M^{i} \cong \Pi_{i=1}^{\infty} M^{i} \cong \Sigma_{E} M^{i}$.

2. Lemmas. We say that a subset $K \subset M$ is infinitely deficient if there exists a homeomorphism $b$ from $M$ to $l_{1} \times M$ such that $b(K) \subset\{0\} \times M$. We say that $K \subset M$ is strongly negligible if, for every open cover $G$ of $M$ there exists a homeomorphism $b$ from $M$ onto $M \backslash K$ such that for any $x \in M$ there is a $g \in G$ which contains both $x$ and $b(x)$.

Lemma 1. Let $M$ be a complete metric space, $K \subset M$ a countable union of closed, infinitely deficient sets. Then $K$ is strongly negligible in $M$. 
Proof. Lemma 1 in Cutler's paper [6] contains this result.

A set $K \subset M$ has property $Z$ (is a $Z$-set) in $M$ if for each nonempty, homotopically trivial, open set $U$ in $M$ it is true that $U \backslash K$ is nonempty and homotopically trivial.

We need the following two lemmas on Z-sets:

Lemma 2. The following are equivalent in $l_{1}$ :

1. $K$ bas property $\mathrm{Z}$ in $l_{1}$,

2. $K$ bas property $Z$ locally in $l_{1}$ (i.e. eacb point of $K$ lies in an open set $\mathcal{O}$ for which $K \cap \mathcal{O}$ bas property $\mathrm{Z}$ in $\Theta$ ).

Proof. See Lemma 1 [4].

Lemma 3. Countable unions of Z-sets are strongly negligible from $l_{1}$.

Proof. See Anderson's paper [2, Theorem 1].

Lemma 4 is a necessary technical lemma:

Lemma 4. Let $M$ be a metric TVS with translation invariant, strictly monotone metric $d$. Then, if $\left\{x_{n}\right\}$ is a sequence in $M$ converging to $x$ and $\left\{t_{n}\right\}$ is a sequence of nonnegative real numbers such that $d\left(t_{n} x_{n}, 0\right)$ converges to $d(t x, 0)$, then $\left\{t_{n} x_{n}\right\}$ converges to $t x$.

Proof. Suppose $\left\{t_{n}\right\}$ does not converge to $t$. Then there exists a subsequence (call it $\left\{t_{n}\right\}$ again) such that

1. $t_{n} \geq t+\epsilon$ for some fixed $\epsilon>0$, all $n$, or

2. $t_{n} \leq t-\epsilon$ for some fixed $\epsilon>0$, all $n$.

But then

1. $d\left(t_{n} x_{n}, 0\right) \geq d\left((t+\epsilon) x_{n}, 0\right) \rightarrow d((t+\epsilon) x, 0)>d(t x, 0)$, or

2. $d\left(t_{n} x_{n}, 0\right) \leq d\left((t-\epsilon) x_{n}, 0\right) \rightarrow d((t-\epsilon) x, 0)<d(t x, 0)$.

Contradiction. Therefore, $\left\{t_{n}\right\}$ converges to $t$, and, hence $\left\{t_{n} x_{n}\right\}$ converges to $t x$.

Lemma 5. Any complete metric TVS $M$ is bomeomorpbic to $\mathbf{R} \times M / \mathbf{R}$ where $\mathbf{R}$ is the real line.

Proof. In Michael's paper [10], Corollary 7.3 says: Let $G$ be a metrizable group, and $H$ be a closed subgroup which is isomorphic to the additive group of a complete, metrizable, locally convex TVS. Then there exists a cross section, i.e. a continuous function $\psi: G / H \rightarrow G$ such that $\phi \circ \psi=$ identity where $\phi: G \rightarrow$ $G / H$ is the canonical map. Identifying $\mathbf{R}$ with a one-dimensional subspace of $M$, we then have the homeomorphism

$$
\text { b: } \begin{aligned}
M & \rightarrow M / \mathbf{R} \times \mathbf{R} \\
x & \rightarrow(\phi(x), x-\psi \circ \phi(x))
\end{aligned}
$$


where $\phi$ is the canonical map, and where $\psi$ is the cross section guaranteed in the above corollary.

Finally, we observe

Lemma 6. Let $M$ be a complete metric TVS with translation invariant, strictly monotone metric d. Then $\Sigma_{l_{1}}(M, d)=\Sigma_{l_{1}}(M, d /(1+d))$.

Proof. The required isomorphism is the identity map. Just use the fact that if $\left\{x_{i}\right\},\left\{y_{i}\right\} \in \Sigma_{l_{1}}(M, d)$ and $\Sigma_{i=1}^{\infty} d\left(x_{i}, y_{i}\right)<1$, then

$$
\frac{d\left(x_{i}, y_{i}\right)}{2} \leq \frac{d\left(x_{i}, y_{i}\right)}{1+d\left(x_{i}, y_{i}\right)} \leq d\left(x_{i}, y_{i}\right), \text { all } i \text {. }
$$

The notion of summability is the same in both spaces. This can be seen by letting $\left\{y_{i}\right\}=0$ above, and applying the inequality to all elements in the sequence whose absolute value is less than 1 .

3. Proofs of Theorems. Note that it is enough to prove Theorem 1 to prove Theorem 0. This follows from the fact that any infinite dimensional Banach space $B$ has an $l_{1}$ factor, i.e. $B \cong l_{1} \times B$. (See Remark 1.) Thus $\Sigma_{l_{1}} B \simeq B \times \Sigma_{l_{1}} B \simeq$ $l_{1} \times B \times \Sigma_{l_{1}} B \simeq l_{1} \times \Sigma_{l_{1}} B$. Also, Theorem 2 can be obtained by replacing $\Sigma_{l_{1}} M$ by $\Sigma_{l_{1}} M^{i}$ in the proof of Theorem 1 , and observing that the map is constructed coordinate-wise.

Proof of Theorem 1. Applying Lemma 6, we will assume that the metric $d$ on $M$ is bounded. We will first prove that $l_{1} \times \Sigma_{l_{1}}{ }^{M}$ is homeomorphic to $M^{\omega}$.

Step 1. $l_{1} \times \Sigma_{l_{1}} M \simeq \Sigma_{l_{1}}\left[\left(\Sigma_{l_{1}} l_{1}\right) \times M\right]$.

Since $l_{1}=\Sigma_{l_{1}}\left(\Sigma_{l_{1}} l_{1}\right), l_{1} \times \Sigma_{l_{1}} M=\Sigma_{l_{1}}\left(\Sigma_{l_{1}} l_{1}\right) \times \Sigma_{l_{1}} M$. But this is just $\Sigma_{l_{1}}\left[\left(\Sigma_{l_{1}} l_{1}\right) \times M\right]$ when we use addition of the metrics, i.e. $d^{\prime}=||+d$ where || is the norm on $\Sigma_{l_{1}} l_{1}$ and $d$ is the metric on $M$. This is the same space when we use $p=\max (||, d)$ as well, since $p \leq d^{\prime} \leq 2 p$. We will use $p$ in the following.

Step 2. $\Sigma_{l_{1}}\left[\left(\Sigma_{l_{1}} l_{1}\right) \times M\right] \cong \Sigma_{l_{1}}\left[\left(\Sigma_{l_{1}} l_{1} \backslash\{0\}\right) \times(M \backslash\{0\})\right]$.

Now

$$
\Sigma_{l_{1}}\left[\left(\Sigma_{l_{1}} l_{1} \backslash\{0\}\right) \times(M \backslash\{0\})\right]=\Sigma_{l_{1}}\left[\left(\Sigma_{l_{1}} l_{1}\right) \times M\right] \backslash\left[\left(U L^{i j}\right) \cup\left(U M^{i}\right)\right]
$$

where

$$
\begin{aligned}
L^{i j} & =\left\{\left\{\xi^{k}\right\} \in \Sigma_{l_{1}}\left[\left(\Sigma_{l_{1}} l_{1}\right) \times M\right]: \pi_{\Sigma}\left(\xi^{i}\right) \text { has jth coordinate } 0\right\}, \\
M^{i} & =\left\{\left\{\xi^{k}\right\} \in \Sigma_{l_{1}}\left[\left(\Sigma_{l_{1}} l_{1}\right) \times M\right]: \pi_{M}\left(\xi^{i}\right)=0\right\} .
\end{aligned}
$$


Here $\pi_{2}$ and $\pi_{M}$ are the projections onto $\Sigma_{l_{1}} l_{1}$ and $M$. Note that the $L^{i j}$ and $M^{i}$ are all closed. They are infinitely deficient since $l_{1} \cong l_{1} \times l_{1}$ and $M \cong l_{1} \times$ $M$. Thus, by Lemma $1,\left[\left(\cup L^{i j}\right) \cup\left(\cup M^{i}\right)\right]$ is strongly negligible in $\Sigma_{l_{1}}\left[\left(\Sigma_{l_{1}} l_{1}\right) \times M\right]$. In particular, we have the required homeomorphism.

Step 3. $\Sigma_{l_{1}}\left[\left(\Sigma_{l_{1}} l_{1} \backslash\{0\}\right) \times(M \backslash\{0\})\right] \cong\left[\left(\Sigma_{l_{1}} l_{1}\right)^{\omega} \backslash\left(\cup l^{i j}\right) \cup\left(U K^{i}\right)\right] \times[M \backslash(0\}]^{\omega}$. The $l^{i j}$ and $K^{i}$ are defined as follows:

$$
l^{i j}=\left\{\left\{\eta^{k}\right\} \in\left[\Sigma_{l_{1}} l_{1}\right]^{\omega}: \pi_{j}\left(\eta^{i}\right)=0\right\}
$$

and

$$
K^{i}=\left\{\left\{r^{k}\right\} \in\left[\Sigma_{l_{1}} l_{1}\right]^{\omega}: \sum_{k=1}^{\infty} \frac{1}{1+\left|r^{k}\right|} \leq i\right\} .
$$

Here, $\pi_{j}$ is projection onto the $j$ th coordinate.

\section{Define}

$$
\begin{gathered}
b: \Sigma_{l_{1}}\left[\left(\Sigma_{l_{1}} l_{1} \backslash\{0\}\right) \times(M \backslash\{0\})\right] \rightarrow\left[\left(\Sigma_{l_{1}} l_{1}\right)^{\omega} \backslash\left(U l^{i j}\right) \cup\left(K^{i}\right)\right] \times[M \backslash\{0\}]^{\omega} \\
\left\{x_{i}\right\} \mapsto\left(t_{1} x_{1}, t_{2} x_{2}, \cdots, t_{n} x_{n}, \cdots\right)
\end{gathered}
$$

where $t_{n} \geq 0$ is chosen such that

$$
p\left(t_{n} x_{n}, 0\right)=\frac{\sum_{i=n}^{\infty} p\left(x_{i}, 0\right)}{p\left(x_{n-1}, 0\right)} .
$$

For $n=1$, the denominator will be, by definition, 1 . Note that $x_{n}$ is actually a pair: $x_{n}=\left\{x_{n}^{l}, x_{n}^{M}\right\}$, with $x_{n}^{l} \in \Sigma_{l_{1}} l_{1}$ and $x_{n}^{M} \in M$. Thus $t_{n} x_{n}=\left\{t_{n} x_{n}^{l}, t_{n} x_{n}^{M}\right\}$.

Since $p$ is strictly monotone and unbounded on rays, a unique such choice exists. The map $b$ is continuous since it is coordinate-wise continuous by Lemma 4.

Define

$$
g:\left[\left(\Sigma_{l_{1}} l_{1}\right) \times M\right]^{\omega} \rightarrow \Sigma_{l_{1}}\left[\left(\Sigma_{l_{1}} l_{1}\right) \times M\right]
$$

by

$$
\left\{z_{i}\right\} \mapsto\left(s_{1} z_{1}, s_{2} z_{2}, \cdots, s_{n} z_{n}, \cdots\right)
$$

where $s_{n} \geq 0$ is chosen such that

$$
p\left(s_{n} z_{n}, 0\right)=\frac{\left[\Pi_{i=1}^{n} p\left(z_{i}, 0\right)\right]}{\left[\Pi_{i=2}^{n+1}\left(1+p\left(z_{i}, 0\right)\right)\right]} .
$$

Again, a unique such point may be chosen.

To show that $g$ is into $\Sigma_{l_{1}}\left[\left(\Sigma_{l_{1}} l_{1}\right) \times M\right]$, observe that 


$$
\sum_{n=1}^{\infty}\left[\frac{\Pi_{i=1}^{n} p\left(z_{i}, 0\right)}{\Pi_{i=2}^{n+1}\left(1+p\left(z_{i}, 0\right)\right)}\right]=p\left(z_{1}, 0\right)\left\{\sum_{n=1}^{\infty}\left[\frac{\Pi_{i=2}^{n} p\left(z_{i}, 0\right)}{\Pi_{i=2}^{n+1}\left(1+p\left(z_{i}, 0\right)\right)}\right]\right\}
$$

Let $r_{i}=d\left(z_{i}, 0\right)$. Then, looking at the partial sums $\delta^{n}$ of the bracket on the right (\{\}), we have by induction

$$
\delta_{n}=1-r_{2} \cdot r_{3} \cdots r_{n} \cdot r_{n+1} /\left(1+r_{2}\right)\left(1+r_{3}\right) \cdots\left(1+r_{n}\right)\left(1+r_{n+1}\right) .
$$

Note that $0<r_{i} /\left(1+r_{i}\right)<1$, all $i$. Therefore, letting $u_{i}=1-r_{i} /\left(1+r_{i}\right)=1 /\left(1+r_{i}\right)$, we have

$$
\begin{aligned}
\left(\frac{r_{2}}{1+r_{2}}\right) \cdots\left(\frac{r_{n+1}}{1+r_{n+1}}\right) & =\left(1-u_{2}\right) \cdots\left(1-u_{n}\right) \\
& \leq \exp \left(-u_{2}-u_{3}-\cdots-u_{n}\right) \text { since } 0<u_{i}<1, \text { all } i, \\
& =\exp \left(-\frac{1}{1+r_{2}}-\cdots-\frac{1}{1+r_{n+1}}\right) .
\end{aligned}
$$

If $\sum_{i=1}^{\infty} 1 /\left(1+r_{i}\right)=\infty$, then $\exp \left(-1 /\left(1+r_{2}\right)-\cdots-1 /\left(1+r_{n+1}\right)\right)$ tends toward 0 . Therefore, the sum in brackets is 1 . If $0<\sum_{i=1}^{\infty} 1 /\left(1+r_{i}\right)<\infty$, then $0<$ $\exp \left(-\Sigma 1 /\left(1+r_{i}\right)\right)<1$. Hence, the sum is less than or equal to $1-$ $\exp \left(-\Sigma 1 /\left(1+r_{i}\right)\right)$. Therefore,

$$
\begin{aligned}
\sum_{n=1}^{\infty}\left[\frac{\Pi_{i=1}^{n} p\left(z_{i}, 0\right)}{\Pi_{i=2}^{\infty}\left(1+p\left(z_{i}, 0\right)\right)}\right] & \leq p\left(z_{1}, 0\right)\left[1-\exp \left(-\sum_{i=2}^{\infty} \frac{1}{1+p\left(z_{i}, 0\right)}\right)\right] \\
& <\infty .
\end{aligned}
$$

By Theorem 15.5 of [11], we also have that $\Pi_{i=2}^{\infty}\left(1-u_{i}\right)>0$ if $\sum_{i=2}^{\infty} u_{i}<\infty$. Now, let $\tilde{M}=\left[\left(\Sigma_{l_{1}} l_{1}\right)^{\omega} \backslash\left(\cup l^{i j}\right) \cup\left(\cup K^{i}\right)\right] \times[M \backslash\{0\}]^{a}$. Then, we can show that

$$
\left.g\right|_{\tilde{M}} \circ b=\text { identity on } \Sigma_{l_{1}}\left[\left(\Sigma_{l_{1}} l_{1} \backslash\{0\}\right) \times(M \backslash\{0\})\right]
$$

by writing out the composition coordinate-wise, and applying induction. Also, $\left.g\right|_{\tilde{M}}$ is continuous since, given $\epsilon>0$ and $\left\{z_{j}\right\}$, choose $N$ such that

$$
\sum_{i=N}^{\infty} p\left(g\left(\left\{z_{j}\right\}\right)_{i}, 0\right)<\frac{\epsilon}{4},
$$

where $g\left(\left\{z_{j}\right\}\right)_{i}$ is the $i$ th coordinate of $g\left(\left\{z_{j}\right\}\right)$. Then pick $N_{i}\left(z_{i}\right)$, a neighborhood of the $i$ th coordinate $(i=1, \ldots, N)$, such that given $\left\{z_{j}^{\prime}\right\}$ with $z_{i}^{\prime} \in N_{i}, i=1,2$, $\cdots, N$, implies

$$
p\left(g\left(\left\{z_{j}\right\}\right)_{i}, g\left(\left\{z_{j}^{\prime}\right\}\right)_{i}\right)<\epsilon / 4 N, \quad i=1, \ldots, N-1,
$$


and, in addition, such that for $N_{1}$, we have $\left|p\left(z_{1}^{\prime}, 0\right)-p\left(z_{1}, 0\right)\right|<\epsilon / 4 N$. Then, given $\left\{z_{j}^{\prime}\right\}$ such that $z_{i}^{\prime} \in N_{i}, i=1, \ldots, N$, implies

$$
\begin{aligned}
\sum_{i=1}^{\infty} p\left(g\left(\left\{z_{j}\right\}\right)_{i}, g\left(\left\{z_{j}^{\prime}\right\}\right)_{i}\right) \\
\quad \leq \sum_{i=1}^{N-1} p\left(g\left(\left\{z_{j}\right\}\right)_{i}, g\left(\left\{z_{j}^{\prime}\right\}\right)_{i}\right)+\sum_{i=N}^{\infty} p\left(g\left(\left\{z_{j}\right\}\right)_{i}, 0\right)+\sum_{i=N}^{\infty} p\left(0, g\left(\left\{z_{j}^{\prime}\right\}\right)_{i}\right) \\
\leq(N-1)(\epsilon / 4 N)+\epsilon / 4+\epsilon / 4+N(\epsilon / 4 N)<\epsilon .
\end{aligned}
$$

This follows from:

$$
\begin{gathered}
\sum_{i=1}^{\infty} p\left(g\left(\left\{z_{j}^{\prime}\right\}\right)_{i}, 0\right)<\sum_{i=1}^{\infty} p\left(g\left(\left\{z_{j}\right\}\right)_{i}, 0\right)+\epsilon / 4 N \\
\| \\
p\left(z_{1}^{\prime}, 0\right) \quad p\left(z_{1}, 0\right)+\epsilon / 4 N .
\end{gathered}
$$

Therefore, $\sum_{i=N}^{\infty} p\left(g\left(\left\{z_{j}^{\prime}\right\}\right)_{i}, 0\right)<\epsilon / 4+N(\epsilon / 4 N)$.

Now, to show that $\left.b \circ g\right|_{\tilde{M}}=$ identity on $\tilde{M}$, we again write out the composition coordinate-wise and apply induction. Thus, we have that $b$ is the required homeomorphism.

Step 4. $\left[\left(\Sigma_{l_{1} l_{1}}\right)^{\omega} \backslash\left(U l^{i j}\right) \cup\left(\cup K^{i}\right)\right] \times[M \backslash(0\}]^{\omega} \simeq\left(\Sigma_{l_{1}} l_{1}\right)^{\omega} \times M^{\omega}$.

We know that $\{0\}$ is strongly negligible from $M$. Therefore $(M \backslash\{0\})^{\omega} \simeq M^{\omega}$. To show

$$
\left.\left[\left(\Sigma_{l_{1}} l_{1}\right)^{\omega}\right)\left(U_{l^{i j}}\right) \cup\left(U_{K^{i}}\right)\right] \simeq\left(\Sigma_{l_{1}} l_{1}\right)^{\omega}
$$

we will show that each $K^{j}$ and $l^{i j}$ is a $Z$-set.

First, we claim each $K^{j}$ is closed. To show this, pick $\left\{x^{k}\right\} \in\left(K^{j}\right)^{c}$ where $\left(K^{j}\right)^{c}$ is the complement of $K^{j}$. Then $\sum_{k=1}^{\infty} 1 /\left(1+\left|x^{k}\right|\right)>j$. Pick $N$ such that $\sum_{k=1}^{N} 1 /\left(1+\left|x^{k}\right|\right)>j$. Then there exists a neighborhood $U$ of $\left\{x^{k}\right\}_{k=1}^{N}, U C$ $\left[\Sigma_{l_{1} l_{1}}\right]^{N}$ such that $\Sigma_{k=1}^{\infty} 1 /\left(1+\left|y^{k}\right|\right)>j$ for all $\left\{y^{k}\right\} \in U$. Then, let $\theta=U \times$ $\Pi_{N_{+}}^{\infty}\left[\Sigma_{l_{1}} l_{1}\right] . \Theta$ is open in $\left[\Sigma_{l_{1}} l_{1}\right]^{\omega}$, and $\left\{x^{k}\right\} \in \Theta$. Also $O \cap K^{j}=\varnothing$. Thus $K^{j}$ is closed. Moreover, $K^{j}$ is nowhere dense since it contains no bounded sequences and is closed, and any open set must contain bounded sequences.

We claim $K^{j}$ is a $Z$-set. Given a convex basic open set $U$ and a map $f: S^{n} \rightarrow$ $\mathcal{U} \backslash K^{i}$, look at $f\left(s^{n}\right)$. For all $f(s) \in \bigcup_{i>j} K^{i}$, pick a constant $N$ such that

(1) $\sum_{i=1}^{N} 1 /\left(1+\left|f(s)_{i}\right|\right)>j$, and

(2) U contains full factors from $N$ on. 
This can be done since $f\left(s^{n}\right)$ is compact and lies in $\left(K^{j}\right)^{c}$. Then to each $f(s) \epsilon$ $f\left(s^{n}\right)$ associate the point $\left\{x_{k}\right\}$ such that $\left(x_{1}, \ldots, x_{N}\right)=\left(f(s)_{1}, \ldots, f(s)_{N}\right)$ and $x_{k}=0$ for all $k>N$. Then, the line segment from $f(s)$ to $\left\{x_{k}\right\}$ is not in $K^{j}$. But then, since $\left(\left[\Sigma_{l_{1}} l_{1}\right]^{\omega}\right)^{\gamma}=\left\{\left\{\eta^{k}\right\} \in\left[\Sigma_{l_{1}} l_{1}\right]^{\omega}: \eta^{i}=0\right.$ for all but a finite number of $\left.i^{\prime} s\right\}$ is contractible in itself, we may define a map $\tilde{f}: E^{n+1} \rightarrow \mathcal{U} \backslash K^{j}$ with $\tilde{f} \mid S^{n}=f$. Thus, by Lemma 2, each $K^{j}$ is a $Z$-set.

To show that each $l^{i j}$ is a $Z$-set, we can use an argument similar to the above. Just keep away from the appropriate 0 coordinate.

Thus, by Lemma $3\left[\left(\bigcup l^{i j}\right) \cup\left(\bigcup K^{i}\right)\right]$ is strongly negligible, and we have the homeomorphism.

Step 5. $\left(\Sigma_{l_{1}} l_{1}\right)^{\omega} \times M^{\omega} \simeq M^{\omega}$.

$\Sigma_{l_{1}} l_{1}=l_{1}$. Therefore $\left(\Sigma_{l_{1}} l_{1}\right)^{\omega} \times M^{\omega}=l_{1}^{\omega} \times M^{\omega}$. By Lemma 5 , we have $M \cong$ $\mathbf{R} \times M / \mathbf{R}$. Thus $M^{\omega} \simeq(\mathbf{R} \times M / \mathbf{R})^{\omega} \simeq \mathbf{R}^{\omega} \times(M / \mathbf{R})^{\omega}$. By Anderson's paper [1] we have $\mathbf{R}^{\omega} \simeq l_{1} \simeq l_{1}^{\omega}$. Thus $M^{\omega} \simeq \mathbf{R}^{\omega} \times(M / \mathbf{R})^{\omega} \simeq \mathbf{R}^{\omega} \times \mathbf{R}^{\omega} \times(M / \mathbf{R})^{\omega} \simeq l_{1} \times$ $(\mathbf{R} \times M / \mathbf{R})^{\omega}=l_{1}^{\omega} \times M^{\omega}$. Thus $M^{\omega} \simeq l_{1}^{\omega} \times M^{\omega} \simeq\left(\Sigma_{l_{1}} l_{1}\right)^{\omega} \times M^{\omega}$.

From Step 1 through Step 5 , we have $l_{1} \times \Sigma_{l_{1}} M \cong M^{\omega}$. Also, Step 5 shows that $M^{\omega} \cong l_{1} \times M^{\omega}$.

If we assume $M \simeq M^{\omega}$, then $M \simeq M^{\omega} \cong l_{1} \times M^{\omega} \cong l_{1} \times M$. Hence $M \cong M^{\omega} \simeq$ $l_{1} \times \Sigma_{l_{1}} M \simeq l_{1} \times M \times \Sigma_{l_{1}} M \simeq M \times \Sigma_{l_{1}} M \simeq \Sigma_{l_{1}} M$.

If we assume $M \cong l_{1} \times M$ and $M \cong \Sigma_{l_{1}} M$, then we have $M \cong \Sigma_{l_{1}} M \cong M \times$ $\Sigma_{l_{1}} M \cong l_{1} \times M \times \Sigma_{\omega_{1}} M \cong l_{1} \times \Sigma_{l_{1}} M \cong M^{\omega}$.

But, then, we have proven Theorem 1.

Proof of Theorem 3. Let $E$ and $E^{\prime}$ be any two of the spaces listed. If $F$ is a coordinate space, we define $F^{+}=\left\{\left\{x^{i}\right\} \in F: x^{i} \geq 0\right.$ for all $\left.i\right\}$. Then, Bessaga has shown, in [5], that there exists a homeomorphism $b: E^{+} \rightarrow\left(E^{\prime}\right)^{+}$such that, for $\left\{x^{i}\right\} \in E^{+}, x^{j}=0$ if and only if $\pi_{j}\left(b\left(\left\{x^{i}\right\}\right)\right)=0 . \pi_{j}$ is projection onto the $j$ th coordinate.

Let $d^{\prime}$ be the max metric on $l_{1} \times M$, and define

$$
\text { H: } \begin{aligned}
\Sigma_{E}\left(l_{1} \times M\right) & \rightarrow \Sigma_{E^{\prime}}\left(l_{1} \times M\right) \\
\left\{x_{i}\right\} & \mapsto\left\{t_{i} x_{i}\right\}
\end{aligned}
$$

where each $t_{i} \geq 0$ is chosen such that $\left\{d^{\prime}\left(t_{i} x_{i}, 0\right)\right\}=b\left(\left\{d^{\prime}\left(x_{i}, 0\right)\right\}\right)$.

Then $H$ is $1-1$ since, if $\left\{t_{i} x_{i}\right\}=H\left(\left\{x_{i}\right\}\right)=H\left(\left\{y_{i}\right\}\right)=\left\{s_{i} y_{i}\right\}$, then $b\left(\left\{d^{\prime}\left(x_{i}, 0\right)\right\}\right)=$ $b\left(\left\{d^{\prime}\left(y_{i}, 0\right)\right\}\right)$. Hence, $d^{\prime}\left(x_{i}, 0\right)=d^{\prime}\left(y_{i}, 0\right)$ for all $i$. But then $\left\{x_{i}\right\}=\left\{y_{i}\right\}$ since $d^{\prime}$ is strictly monotone.

$H$ is onto since, given $\left\{y_{i}\right\} \in \Sigma_{E^{\prime}}\left(l_{1} \times M\right)$, choose $\left\{s_{i} y_{i}\right\}$ such that $\left\{d^{\prime}\left(s_{i} y_{i}, 0\right)\right\}=b^{-1}\left(\left\{d^{\prime}\left(y_{i}, 0\right)\right\}\right)$. Then $H\left(\left\{s_{i} y_{i}\right\}\right)=\left\{y_{i}\right\}$ since $d^{\prime}$ is strictly monotone. This also shows how to define $H^{-1}$. 
Now $H$ and $H^{-1}$ are continuous by Lemma 4. Note that the same proof works if we allow $M$ to change.

\section{REFERENCES}

1. R. D. Anderson, Hilbert space is homeomorphic to the countable infinite product of lines, Bull. Amer. Math. Soc. 72 (1966), 515-519. MR 32 \#8298.

2. - Strongly negligible sets in Fréchet manifolds, Bull. Amer. Math. Soc. 75 (1969), 64-67. MR 38 \#6634.

3. R. D. Anderson and R. H. Bing, A complete elementary proof that Hilbert space is homeomorphic to the countable infinite product of lines, Bull. Amer. Math. Soc. 74 (1968), 771-792. MR 37 \#5847.

4. R. D. Anderson, D. W. Henderson and J. E. West, Negligible subsets of infinitedimensional manifolds, Compositio Math. 21 (1969), 143-150. MR 39 \#7630.

5. C. Bessaga, On topological classification of complete linear metric spaces, Fund. Math. 56 (1964/65), 251-288. MR 31 \#2580.

6. W. H. Cutler, Negligible subsets of infinite-dimensional Fréchet manifolds, Proc. Amer. Math. Soc. 23 (1969), 668-675. MR 40 \#2133.

7. M. Eidelheit and S. Mazur, Eine Bemerkung über die Räume vom Typus (F), Studia Math. 7 (1938), 159-161.

8. D. W. Henderson and R. Schori, Topological classification of infinite dimensional manifolds by homotopy type, Bull. Amer. Math. Soc. 76 (1970), 121-124. MR 40 \#4976.

9. J. L. Kelley and I. Namioka, et al., Linear topological spaces, University Series in Higher Math., Van Nostrand, Princeton, N. J., 1963. MR 29 \#3851.

10. E. Michael, Convex structures and continuous selections, Canad. J. Math. 11 (1959), 556-575. MR 22 \#230.

11. W. Rudin, Real and complex analysis, McGraw-Hill, New York, 1966. MR 35 $\# 1420$.

DEPARTMENT OF MATHEMATICS, CORNELL UNIVERSITY, ITHACA, NEW YORK 14850 